\title{
Frustration-mediated learning of a barpress response
}

\author{
PETER C. SENKOWSKI \\ Northern Illinois University, DeKalb, Illinois 60115 \\ and \\ M. RAY DENNY \\ Michigan State University, East Lansing, Michigan 48823
}

\begin{abstract}
Following 72 reinforced placements in a box, rats received 30 additional nonreinforced placements during which time a bar was initially available. Under one condition a barpress response resulted in the rat's being immediately removed from the box. Under the other condition, a barpress resulted in removal of the bar from the box while the rat remained in the box for a fixed period of time. Barpress speeds of both groups, but especially of the latter, improved with trials, whereas the slower speeds of control subjects, which received nonreinforced placements prior to barpress testing, showed no evidence of learning. The results were interpreted as supporting a prediction from elicitation theory that frustration can mediate learning through consistent elicitation of a response that is characteristic of frustration conditions, even when reinforcement in the form of frustration reduction is absent.
\end{abstract}

According to frustration theory (Amsel, 1958, 1962), when nonreward occurs in the presence of stimuli which have consistently accompanied reward in the past, a primary emotional or motivational response of frustration $\left(R_{F}\right)$ results. In addition to having energizing properties, $R_{F}$, when it undergoes reduction, is assumed to have reinforcing properties so that responses which precede this event should be learned. This assumption has been tested by Wagner (1963) and extensively by Daly (e.g., Daly, 1969a, $1969 \mathrm{~b}, 1974)$; but the results of these studies, while generally consistent with predictions based on frustration theory, have not ruled out alternative interpretations.

Daly and McCroskery (1973) have reported the results of two experiments which seem to provide strong evidence in support of the notion that frustration reduction can reinforce behaviors other than simply locomotion away from the source of frustration, as was studied in the hurdle-jump experiments by Daly and by Wagner cited above. Daly and McCroskery found, in terms of a progressive decline in latency, that rats learned to press a bar if this response resulted in their being removed from a box in which they had been consistently fed but which no longer contained food and could now be assumed to arouse $R_{F}$. They interpreted their results as in-

Parts of this research were carried out while the second author was a visiting professor at Northern Illinois University. Reprint requests should be sent to Peter $C$. Senkowski, Department of Psychology, Northern Illinois University, DeKalb, Illinois 60115. dicating the reinforcing value of frustration reduction, or escape from frustration, in the learning of a barpress response.

An alternative to the frustration-reduction position stems directly from elicitation theory (Denny, 1970, 1971a, 1971b; Denny \& Adelman, 1955). In fact the frustration-elicitation interpretation was used by Adelman and Maatsch (1956) to explain one of the earliest observations of frustrationmediated learning of a jump-out response. This interpretation, however, was immediately translated by others into learning through frustration reduction, as recently discussed by Denny (1971b). According to the elicitation framework, the barpress response is learned in the Daly and McCroskery study because it is rather consistently elicited by frustration (empty food cup) in simple contiguity with the box stimuli, including the presence of the bar. The barpress, as part of a class of unconditioned attack behaviors characteristically elicited by frustration, is classically conditioned to the concurrent stimulus situation. In the stimulus situation used by Daly and McCroskery (1973), the bar is a relatively prominent component and the most obvious and available object to attack.

According to elicitation theory, or a Guthrian point of view, either the removal of the subject or the removal of the bar from the box following a barpress response prevents the subject from making a different or alternative response in the presence of the bar, which of course prevents the learning of an alternative response to the bar and protects any learned association between the stimulus complex 
containing the bar and the barpress response. That is, alternative responses that occur subsequent to the initial barpress would occur in a different stimulus situation, namely, outside the box or in the box minus the bar, and would therefore not interfere with barpress learning.

The present experiment was designed to compare the alternative interpretations outlined above. If the frustration-reduction explanation is correct, then learning in the experimental situation described by Daly and McCroskery (1973) should occur only if the change in the stimulus situation following a barpress involves the elimination of frustration-eliciting cues. The alternative contiguity interpretation requires only that no response other than the barpress occur consistently in contiguity with the bar in the box. Two groups, experimental and control, essentially replicated Experiment II by Daly and McCroskery (1973), in that subjects were taken out of the box contingent on a response. Two other groups, experimental and control, tested the contiguity interpretation by taking the bar out of the apparatus contingent on a response while leaving the subject in the apparatus for a fixed period of time. One additional bar-out group was employed to test for possible effects of initial familiarity with the bar on subsequent barpress performance. Another way of viewing the bar-out groups in the present experiment is that they constitute a critical control that Daly and McCroskery did not run.

\section{METHOD}

\section{Subjects}

Sixty naive male hooded rats, $95-110$ days old at the start of the experiment, were obtained from a colony maintained by the Psychology Department at Northern Illinois University. The subjects were reduced to $80 \%$ of their ad-lib weights through restricted feeding, and maintained at this level throughout the experiment. Water was continuously available except when the subjects were in the experimental apparatus.

\footnotetext{
Apparatus

The apparatus consisted of one compartment of a twocompartment hurdle-jumping apparatus patterned after that of Daly (1969b). The compartment used was constructed of plywood with inside dimensions of $28 \times 11 \times 14 \mathrm{~cm}$, and had a solid wooden floor. The interior was painted flat white and the walls were lined with clear Plexiglas. A guillotine door, resting on a hurdle, was included in one of the $11-\mathrm{cm}$-wide walls. This door remained closed throughout the experiment. A $2.54-\mathrm{cm}$-square metal food cup was mounted $4 \mathrm{~cm}$ above the floor on one of the $28-\mathrm{cm}$ walls, $3.5 \mathrm{~cm}$ from the door wall. A $1.27-\mathrm{cm}$-diam hole, $4.5 \mathrm{~cm}$ above the floor and $7.5 \mathrm{~cm}$ from the door wall, allowed a Lehigh Valley omnidirectional lever to be inserted $2.54 \mathrm{~cm}$ into the compartment. This hole was filled with a dowel, which fit flush with the inside of the wall, when the bar was absent. Placement of the subject into the compartment broke a photobeam $4 \mathrm{~cm}$ above the floor and $9 \mathrm{~cm}$ from the door wall, resulting in the starting of a Hunter Model 120C Klockounter $(.01 \mathrm{sec})$. When the subject pressed the bar, the Klockounter stopped. A box of the same dimensions as the compartment was used as a cover and to supply illumination in the form of a $7.5-\mathrm{W}$ lamp mounted above a sheet of sanded Plexiglas.
}

\section{Procedure}

Prior to the start of deprivation, the subjects were assigned in equal numbers to five treatment conditions. Group assignment was according to a randomized block procedure based on ad-lib body weights. Throughout the experiment, the subjects were run in pairs with both members of a pair assigned to the same group. The intertrial interval, timed with a stopwatch, was never less than 1 min but was sometimes slightly longer, depending on how much time was required for the subject either to consume the pellets (training phase) or press the bar (testing phase). Days 1 through 6 constituted the training phase. On each of the 12 daily trials given during this phase, the subjects were placed in the compartment directly over the food cup. For subjects assigned to Groups BO (bar-out), SO (subject-out), and BP (bar present during training), the food cup was baited on each trial with 12 .045 -g Noyes pellets. When all the pellets had been consumed and at least $60 \mathrm{sec}$ had elapsed, the subject was removed from the apparatus to a holding cage equipped with a water bottle while its running mate received a trial. Subjects typically required slightly more than $60 \mathrm{sec}$ to eat all of the pellets. For subjects in control groups SOC and BOC, the food cup was not baited during placements and the subjects remained in the apparatus for $60 \mathrm{sec}$ on each trial. During the training phase, the bar was present in the apparatus on every trial for subjects in group BP, but was not functional. For all other groups, the bar was absent.

The barpress testing phase took place on Days 7 and 8 . On each of the 15 daily test trials, all subjects were placed in the apparatus with the bar present and the food cup empty. For subjects in Groups SO and SOC, a barpress resulted in their immediate removal by hand from the apparatus to the holding cage. For subjects in Groups BO, BOC, and BP, a barpress resulted in the bar's being immediately removed from the apparatus while the subject remained in the compartment for a total of $60 \mathrm{sec}$ or for $10 \mathrm{sec}$ following a barpress, whichever yielded the longer total time. Barpress latencies were recorded on each trial. If a subject failed to respond within $60 \mathrm{sec}$, it was removed from the apparatus to the holding cage and a latency of $60 \mathrm{sec}$ was recorded.

\section{RESULTS AND DISCUSSION}

Mean barpress speeds (1/latency in sec) in blocks of three trials are presented in Figure 1. Separate analyses were conducted on the data obtained from the subject-removed groups (SO and SOC) and barremoved groups (BO, BOC, and $\mathrm{BP}$ ). Barpress speeds of the subject-removed groups are shown in the left half of Figure 1. An analysis of variance of these data (two groups by 10 trial blocks) resulted in a reliable Groups by Trial Blocks interaction, $F(9,198)=2.39, p<.025$. Subsequent tests of simple main effects yielded significant differences between groups SO and SOC on Trial Blocks 3 and 4 (ps<.025). To determine whether learning had occurred in either of these groups, Treatment by Subjects analyses of variance were conducted using the data from each group. A reliable Trial Blocks effect, $F(9,99)=3.67, p<.001$, was obtained only for group SO. The results of these analyses in conjunction with the visual impression given in the left half of Figure 1 indicate that, while Group SO demonstrated both acquisition and extinction of the barpress response, subjects in the nonfrustrated control group (SOC) did not. The performance of Groups SO and SOC on the first day of testing 


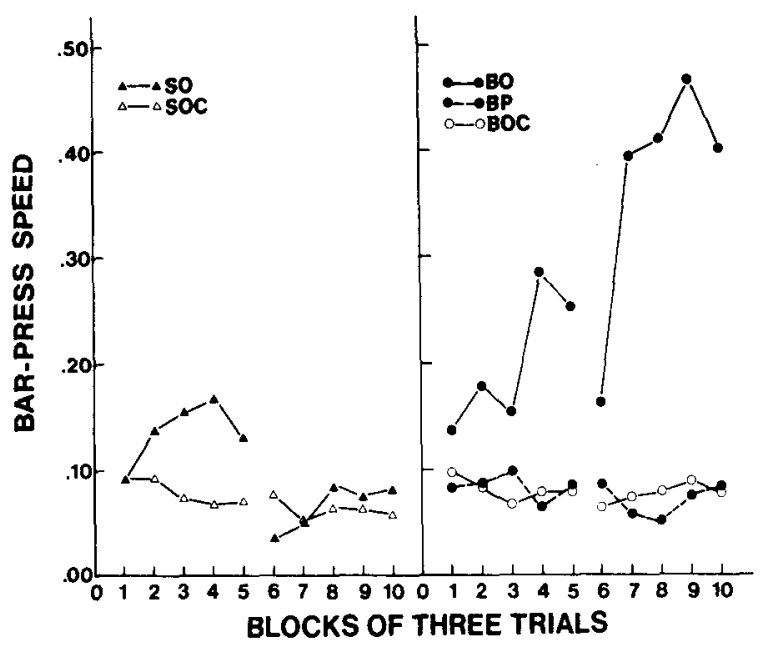

Figure 1. Mean barpress speed (reciprocal of latency) for all groups as a function of blocks of three test trials. The left half of the figure shows barpress speeds for subjects which were removed from the apparatus contingent on a response, while the right half depicts performance of subjects for which the bar was removed from the apparatus contingent on a response.

replicated in all essential respects that of similar groups in Experiment II by Daly and McCroskery (1973), including absolute levels of performance, and lend further support to the conclusion that frustration can serve to energize performance of a barpress response.

The barpress speeds in the bar-removed conditions are shown as a function of trial blocks in the right half of Figure 1. An analysis of variance ( 3 by 10) of these data resulted in a reliable Groups by Trial Blocks interaction, $\mathrm{F}(18,297)=2.30, \mathrm{p}<.005$. Further analysis of this interaction revealed no differences between groups on the first block of trials, $F<1$, and marginally reliable differences on Trial Blocks 2, 3, and $5(.05<\mathrm{p}<.10)$. The effect of Groups on Trial Blocks 4, 7, 8, 9, and 10 was significant, with the differences being reliable beyond the .005 level in all cases except on Trial Block 4. Here the difference in barpress speeds between groups was significant at the .05 level. In each comparison, it was found that group BO had reliably faster speeds than did either Group BOC or Group BP. The speeds of the latter two groups were never reliably different. Treatment by Subjects analyses of variance performed on the data from each of these three groups indicated a reliable Trial Blocks effect only for Group BO, $F(9,99)=2.63$, $\mathrm{p}<.025$, supporting the visual impression given in the right half of Figure 1 that only subjects in this group learned the barpress response.

The most important finding of the present experiment is that both Group SO and Group BO learned the barpress response. This suggests that reinforcement in the form of frustration reduction was not necessary for learning, since frustration reduction following a barpress presumably occurred only in Group SO. Repeated pairings of the bar with frustrative nonreward in Group BO might be expected to result in the bar's becoming aversive so that its removal could be considered reinforcing, but a considerable number of pairings would presumably be necessary before this could happen. An examination of the right half of Figure 1 suggests that the superiority of Group BO over BOC was present from the beginning of testing.

When the argument offered above is coupled with the fact that Group BO's performance was superior to that of Group SO, it becomes more difficult to adhere to a frustration-reduction interpretation of the present data. An analysis of variance comparing those two groups, using blocks of test trials as a within-subjects factor, resulted in a reliable Groups by Trial Blocks interaction, $F(9,198)=3.05$, $\mathrm{p}<.005$. Subsequent tests for simple effects indicated that subjects in group BO pressed the bar faster than did those in Group SO on Trial Blocks 7, 8,9 , and 10 , ps $<.01$ in each case. If barpressing in these groups were being reinforced via frustration reduction, the performance of Group SO should be superior to that of Group BO, since a barpress in Group SO resulted in immediate and complete removal of frustration-related stimuli while removal of the bar in the BO condition constituted little, if any, reduction of frustration.

A more parsimonious explanation of the present findings is that the barpress response was learned by Groups BO and SO because that response was consistently elicited by frustration in the presence of the stimuli in the situation. Increases in the speed of barpressing over trials would be expected as more of these stimuli came to be associated with the response through contiguity.

Additional, indirect support for the interpretation that what is learned depends upon what response is consistently elicited comes from pilot work which preceded the present experiment. A box with a ceiling $27 \mathrm{~cm}$ high was used, and no barpress learning occurred in either the subject-removed or barremoved conditions. Barpressing rarely occurred, and when it did, it was of an accidental sort as the rat fell against the bar in its attempts to jump out of the box. With the high ceiling, the rat was able to stand on its hind legs and attempt escape. Under those conditions, escape was prepotent over attack; attempts to escape, rather than attacking the bar, were elicited and presumably learned, as observed incidentally.

The superiority of Group BO's performance relative to that of Group SO, though not directly predictable from elicitation theory, is not inconsistent with a contiguity interpretation. The bar could be withdrawn more quickly following a bar- 
press than the subject could be picked up and removed. This means that the change in the stimulus situation following a response was more immediate for Group BO subjects than for those in Group SO, and might be expected to produce better learning in the former group. Another possible explanation of Group BO's superiority is that the handling received by Group SO subjects immediately after a barpress was to some degree aversive, tending to inhibit barpressing somewhat.

Another aspect of the data which requires discussion is the failure of subjects in Group BP to learn the barpress response. The BP condition was identical to BO, with the exception that the bar had been present throughout the direct placement phase of the experiment for BP subjects. Over the first three test trials, each of the subjects in Group BP made at least two barpresses. This would seem to indicate that failure to learn the response in that group cannot be attributed to the subjects' not having experienced the effects of a barpress. The fact that Group BO learned to barpress while Group BP did not suggests that novel stimuli are more likely to be the target of frustration-elicited attack behaviors than are stimuli to which subjects have had an opportunity to habituate. When Group BP subjects were frustrated, the resulting aggressive behaviors tended to be directed toward the stimulus environment in general (e.g., attacks on the closed hurdle door). Group BO subjects, on the other hand, focused on the novel stimulus of the bar for their aggressive behaviors.

The present results support the hypothesis that frustration can rather consistently elicit barpressing behavior as a form of attack and can thereby mediate the learning of such behavior. The results seem to indicate that, while reinforcement through frustration reduction was not ruled out as a possible basis for learning, frustration reduction was not necessary for learning to occur in the present situation.

\section{REFERENCES}

Adelman, H. M., \& MaAtsch, J. L. Learning and extinction based upon frustration, food reward, and exploratory tendency. Journal of Experimental Psychology, 1956, 52, 311-315.

AMSEL, A. The role of frustrative nonreward in noncontinuous reward situations. Psychological Bulletin. 1958, 55, 102-119.

AMSEL, A. Frustrative nonreward in partial reinforcement and discrimination learning: Some recent history and a theoretical extension. Psychological Review, 1962, 69, 306-328.

DALY. H. B. Learning of a hurdle-jump response to escape cues paired with reduced reward or frustrative nonreward. Journal of Experimental Psychology, 1969. 79. 146-157. (a)

DALY, H. B. Is instrumental responding necessary for nonreward following reward to be frustrating? Journal of Experimental Psychology, 1969, 80, 186-187. (b)

DALY, H. B. Reinforcing properties of escape from frustration aroused in various learning situations. In G. H. Bower (Ed.), The psychology of learning and motivation (Vol. 8). New York: Academic Press, 1974.

Daly. H. B. \& \& McCroskery, J. H. Acquisition of a bar-press response to escape frustrative nonreward and reduced reward. Journal of Experimental Psychology, 1973, 98. 109-112.

DENNY, M. R. Elicitation theory applied to an analysis of the overlearning reversal effect. In J. H. Reynierse (Ed.), Current issues in animal learning. Lincoln, Nebr: University of Nebraska Press. 1970.

Denny, M. R. Relaxation theory and experiments. In F. R. Brush (Ed.). Aversive conditioning and leaming. New York: Academic Press, 1971. (a)

DENNY, M. R. A theory of experimental extinction and its relation to a general theory. In H. H. Kendler \& J. T. Spence (Eds.). Essays in neobehaviorism: A memorial volume to Kenneth $W$. Spence. New York: Academic Press. 1971. (b)

Denny, M. R.. \& Adelman, H. M. Elicitation theory: I. An analysis of two typical learning situations. Psychological Review, 1955. 62. 290-296.

W AGNER. A. R. Conditioned frustration as a learned drive. Journal of Experimental Psychology, 1963. 66. 142-148.

(Received for publication February 4, 1977; revision accepted June 3,1977 .) 